\title{
Dispersion of hyperenhancement in late gadolinium enhancement cardiovascular magnetic resonance measured with Moran's I is associated with a decrement in LVEF 6 months after cardiotoxic chemotherapy
}

\author{
Jennifer H Jordan ${ }^{1 *}$, Craig A Hamilton ${ }^{2}$, Ralph B D’Agostino ${ }^{3}$, Julia Lawrence ${ }^{4}$, Sujethra Vasu', \\ W Gregory Hundley ${ }^{1,5}$
}

From 16th Annual SCMR Scientific Sessions

San Francisco, CA, USA. 31 January - 3 February 2013

\section{Background}

In animals and human subjects, an increase in background signal intensity observed on late gadolinium enhanced (LGE-SI) images is associated with a decrement in left ventricular ejection fraction (LVEF) during receipt of anthracycline chemotherapy. Moran's I statistic is a measurement of spatial dispersion of hyperenhanced voxels relative to the mean myocardial LGE-SI, ranging from highly clustered ( $\mathrm{I}=+1)$ to highly diffuse ( $\mathrm{I}=-1)$ (Figure 1 ). We hypothesize that a change in the distribution of hyperenhanced voxels (due to the development of high signal "micro clusters") is associated with a decrement in LVEF after cardiotoxic chemotherapy.

\section{Methods}

We performed a prospective, extramurally-funded longitudinal cohort study of 51 participants ( 43 women, 8 men; aged $52 \pm 2$ years) scheduled to receive 3 to 4 months of potentially cardiotoxic chemotherapy (anthracycline or trastuzumab) for treatment of breast cancer or hematologic malignancy. Before and then 3 and 6 months after chemotherapy initiation, participants underwent cardiovascular magnetic resonance (CMR) assessments of LVEF, LGE-SI, and Moran's I statistic determined by personnel blinded to participant identifiers and all other aspects of the analyses. Results were analyzed using

IInternal Medicine (Cardiology), Wake Forest University School of Medicine, Winston Salem, NC, USA

Full list of author information is available at the end of the article paired Student's t-tests to test for a difference between baseline and subsequent examinations, and one-way ANOVA to test for trending change. All values are reported as mean \pm standard deviation with $\mathrm{p}$-values $<0.05$ considered statistically signficant.

\section{Results}

37 participants were treated for breast cancer and 14 for hematologic malignancy. A declining LVEF from baseline $(58 \pm 6 \%)$ was observed three months $(54 \pm 7 \%)$ and six months $(53 \pm 7 \%)$ after beginning chemotherapy $(\mathrm{p}<0.0001$ for trend, Figure 2A). Mean LGE-SI, reflecting a change in myocardial $\mathrm{T} 1$ relaxation, increased from $14.0 \pm 5.5$ at baseline to $16.1 \pm 7.6$ three months after starting chemotherapy ( $p=0.03$, Figure $2 B$ ) and remained elevated at 6 months $(15.7 \pm 6.8, \mathrm{p}=0.07$ from baseline $)$. At baseline and 3 months, the patterns of LGE-SI hyperenhancement (Moran's I statistic) showed random distribution $(-0.02 \pm 0.02$ and $-0.02 \pm 0.01$, respectively; $\mathrm{p}=0.91$ ). Six months after chemotherapy initiation, myocardial LGE-SI hyperenhanced voxels became more diffusely distributed as shown in Figure $2 \mathrm{C}(\mathrm{I}=-0.12 \pm 0.14$, $\mathrm{p}<0.001)$.

\section{Conclusions}

We observed that, six months after receipt of chemotherapy, increased late gadolinium enhancement signal intensity (LGE-SI) occurs in a diffusely distributed pattern within the myocardium concurrent with a 


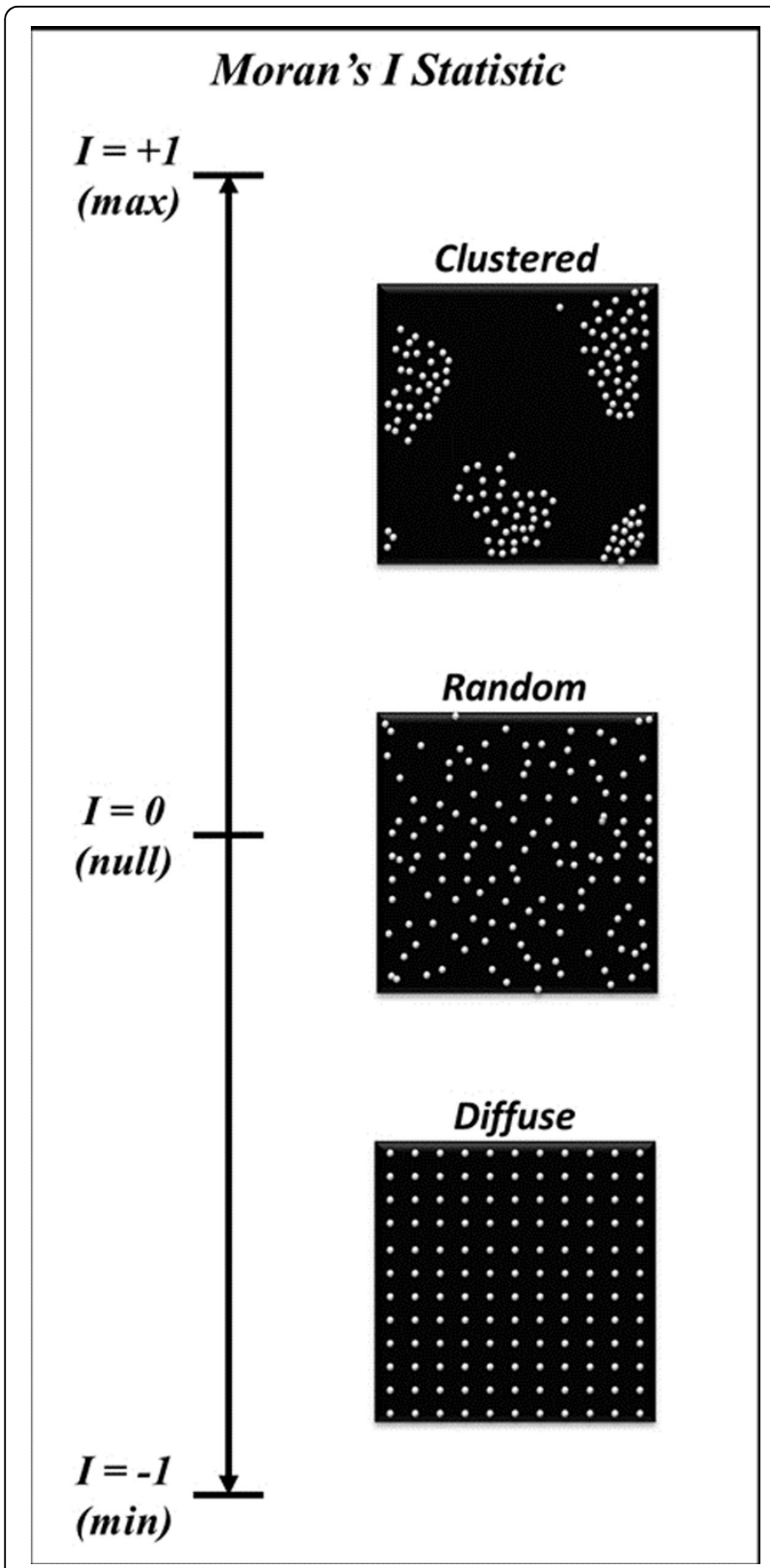

Figure 1 Illustration of the spatial autocorrelation statistic, Moran's I, which describes the distribution of values in a volume in relation to their difference from the mean value and proximity to other voxels of similar intensity value. Moran's I ranges from I=-1 (diffuse distribution) to $\mathrm{I}=+1$ (clustered distribution) and $\mathrm{I}=0$ represents random distribution in the volume of values different from the mean value. Each illustration in the image contains the same number of white dots (or mean value) but different patterns of distribution.

declining LVEF. Moran's I statistic is a novel method to discriminate processes related to a diffuse increase in myocardial T1 (fibrosis, edema) from those related to a clustered increase in myocardial T1 (infarct); further investigations are warranted to study the utility of Moran's I statistic with $\mathrm{T} 1$ and $\mathrm{T} 2$ mapping.

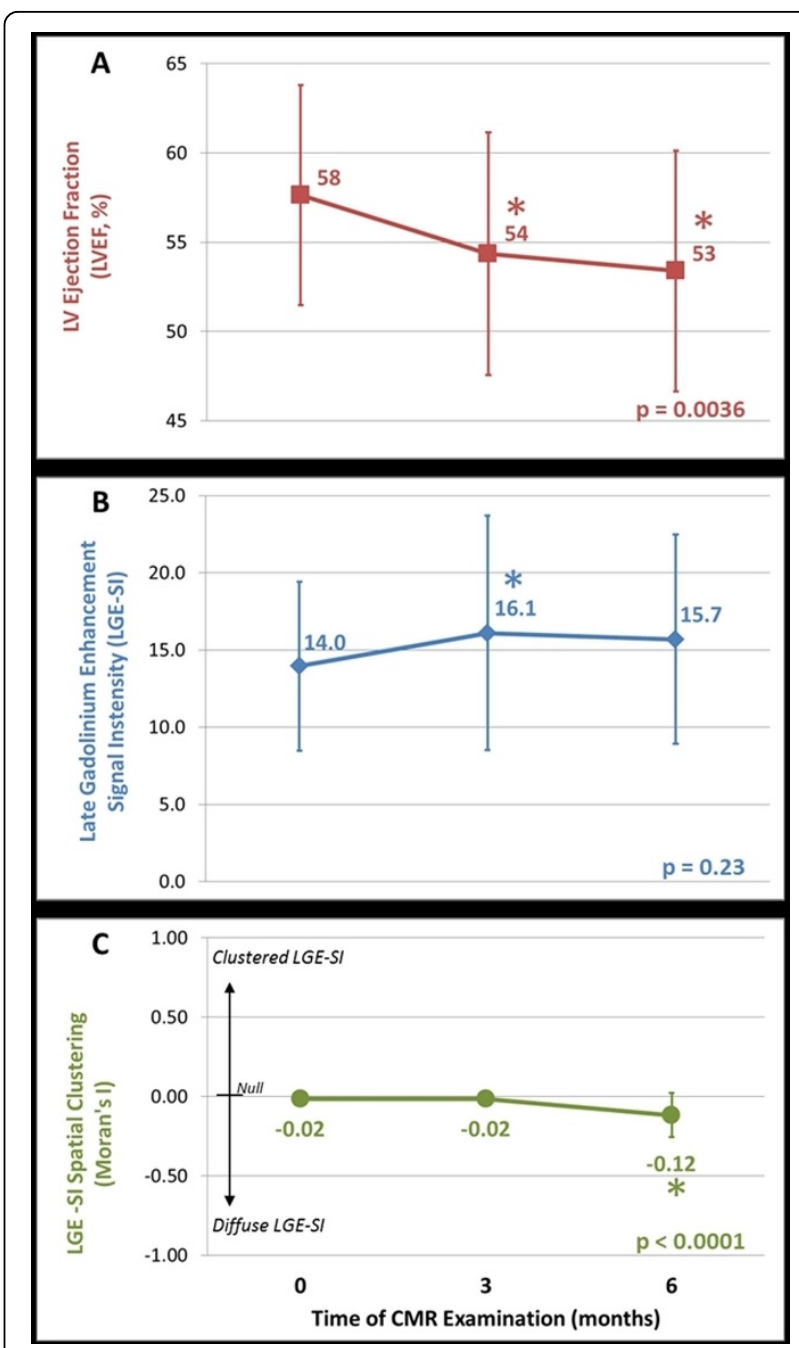

Figure $\mathbf{2}$ Longitudinal measurements of left ventricular ejection fraction (LVEF, Panel A), late gadolinium enhancement signal intensity (LGE-SI, Panel B), and LGE-SI pattern (Moran's I, Panel C) measured with 1.5T cardiovascular magnetic resonance prior to chemotherapy administration (month 0 ) and again 3 and 6 months after administration of cardiotoxic chemotherapy. Mean values \pm standard deviation shown in each panel. Significant differences from baseline by paired t-tests with $p$-values $<0.05$ indicated by $(*)$; $p$-value listed in each panel is for analysis of trend using one-way ANOVA.

\section{Funding}

This work was supported in part by the National Institutes of Health grant R33CA12196 (Hundley), American Heart Association Predoctoral Fellowship 09PRE2210050 (Jordan), and a grant from the Susan G. Komen Foundation BCTR07007769 (Hundley).

\section{Author details}

'Internal Medicine (Cardiology), Wake Forest University School of Medicine, Winston Salem, NC, USA. ${ }^{2}$ Biomedical Engineering, Wake Forest University School of Medicine, Winston-Salem, NC, USA. ${ }^{3}$ Public Health Sciences, Wake Forest University School of Medicine, Winston Salem, NC, USA. ${ }^{4}$ Internal 
Medicine (Hematology and Oncology), Wake Forest University School of Medicine, Winston Salem, NC, USA. ${ }^{5}$ Radiology, Wake Forest University

School of Medicine, Winston Salem, NC, USA.

Published: 30 January 2013

doi:10.1186/1532-429X-15-S1-P156

Cite this article as: Jordan et al:. Dispersion of hyperenhancement in late gadolinium enhancement cardiovascular magnetic resonance measured with Moran's I is associated with a decrement in LVEF

6 months after cardiotoxic chemotherapy. Journal of Cardiovascular Magnetic Resonance 2013 15(Suppl 1):P156.

Submit your next manuscript to BioMed Central and take full advantage of:

- Convenient online submission

- Thorough peer review

- No space constraints or color figure charges

- Immediate publication on acceptance

- Inclusion in PubMed, CAS, Scopus and Google Scholar

- Research which is freely available for redistribution

Submit your manuscript at www.biomedcentral.com/submit 